Methods: In January 2016, the Autoimmune Diseases Study Group (GEASSEMI) created a national registry (SARCOGEAS) of patients with sarcoidosis. Sarcoidosis was diagnosed in agreement with the criteria proposed by the ATS/ERS/WASOG 1999 statement, and extrathoracic disease was classified with the 2014 WASOG instrument.

Results: The cohort consisted of 1082 patients ( $82 \%$ biopsy-proven), including $618(57 \%)$ women and $464(43 \%)$ men, with a mean age at diagnosis of 47yrs; 140 (13\%) patients were born outside Spain, $965(89 \%)$ were White, 69 (6\%) Hispanic, $30(3 \%)$ Black/African American and $18(2 \%)$ Asian. Thoracic involvement was present at diagnosis in $979(90 \%)$ patients, including $437(40 \%)$ patients with stage I, $374(35 \%)$ with stage II, $123(11 \%)$ with stage III and 26 $(2 \%)$ with stage IV. The most frequently reported extrathoracic involvements at diagnosis were cutaneous in $385(36 \%)$ patients, extrathoracic lymph nodes in $218(20 \%)$, liver involvement in $151(14 \%)$ and ocular involvement in $118(11 \%)$. Potentially life-threatening WASOG involvements were reported in frequencies less than $10 \%$, including neurological involvement in $77(7 \%)$ patients, kidney involvement in $59(5 \%)$ or cardiac involvement in $21(2 \%)$. Therapeutic approaches at diagnosis included the use of oral glucocorticosteroids in $637(59 \%)$ patients, immunosuppressive agents in 84 ( $8 \%$, mainly methotrexate in 63 patients) and biological agents in 15 (1\%, mainly infliximab in 10 cases).

Conclusions: In this large series of sarcoidosis from Southern Europe, clinical presentation is dominated by adenopathies (both thoracic and extrathoracic) and cutaneous involvement (erythema nodosum), with lower frequencies in the main extrathoracic involvements than that reported in US and Japanese series.

Disclosure of Interest: None declared

DOI: 10.1136/annrheumdis-2017-eular.4387

\section{THU0540 EFFICACY AND SAFETY OF ADALIMUMAB IN BEHÇET'S DISEASE RELATED UVEITIS: A MULTICENTER RETROSPECTIVE OBSERVATIONAL STUDY}

C. Fabiani ${ }^{1}$, A. Vitale ${ }^{2}$, G. Emmi ${ }^{3}$, L. Vannozzi ${ }^{4}$, G. Lopalco ${ }^{5}$, S. Guerriero ${ }^{5}$, I. Orlando ${ }^{2}$, R. Franceschini ${ }^{6}$, D. Bacherini ${ }^{4}$, L. Cimino ${ }^{7}$, A. Soriano ${ }^{8}$,

B. Frediani ${ }^{2}$, M. Galeazzi ${ }^{2}$, F. Iannone ${ }^{9}$, C. Salvarani ${ }^{10}$, L. Cantarini ${ }^{2}$

${ }^{1}$ Department of Ophthalmology, Humanitas Research Hospital, Milano; ${ }^{2}$ Unit of Rheumatology, University of Siena, Siena; ${ }^{3}$ Department of Experimental and Clinical Medicine; ${ }^{4}$ Department of Surgery and Translational Medicine, Eye Clinic, University of Firenze, Firenze; ${ }^{5}$ Interdisciplinary Department of Medicine, Rheumatology Unit, University of Bari, Bari; ${ }^{6}$ Ophthalmology and Neurosurgery Department, University of Siena, Siena; ${ }^{7}$ Department of Ophthalmology, Arcispedale Santa Maria Nuova IRCCS; ${ }^{8}$ Department of Rheumatology, Arcispedale Santa Maria Nuova IRCCS, Reggio Emilia, Italy, Reggio Emilia; ${ }^{9}$ Unit of Rheumatology, University of Bari, Bari; ${ }^{10}$ Department of Rheumatology, Arcispedale Santa Maria Nuova IRCCS, Reggio Emilia, Italy

Background: Current information on the use of adalimumab (ADA) in the treatment of ocular Behçet's disease (BD) is still based mainly on small series or case reports; nonetheless preliminary evidence is promising.

Objectives: The study aim was to evaluate the efficacy of ADA in a large series of BD-related uveitis.

Methods: We performed a multicenter retrospective observational study including 40 selected patients (66 eyes) receiving ADA. Clinical data were retrospectively analyzed at baseline, at 3 and 12 months of treatment. Primary end-point was: reduction of ocular inflammatory flares. Secondary end-points were: improvement of Best Corrected Visual Acuity (BCVA), reduction of macular thickness measured by optical coherence tomography (OCT), reduction in the occurrence of vasculitis assessed by fluorescein angiography (FA), evaluation of statistically significant differences between patients treated with ADA monotherapy and those undergoing ADA plus DMARDs and in patients firstly treated with ADA compared to patients previously administered with other biologics; ADA steroid sparing effect was also evaluated.

Results: During the first 12 months of ADA therapy the number of flares significantly decreased from 200 flares/100 patients/year to 8.5 flares/100 patients/year $(p<0.0001)$. Similarly BCVA improved if compared to baseline (7.4 \pm 2.9 versus $8.5 \pm 2.1, p=0.03$ ). OCT findings significantly improved showing a mean reduction of central macular thickness (CMT) of $27.27 \pm 42.8$ microns at the end of follow up $(p<0.006)$. FA identified retinal vasculitis in 22 cases at baseline $(55 \%), 8(20 \%)$ cases after 3 months and in only one $(2.5 \%)$ case at 12 -month follow-up. FA improvement was highly significant at 3 and 12 -month follow-up if compared to baseline ( $p<0.0001$ and $p=0.006$, respectively).

Conclusions: ADA is highly effective and safe for the treatment of BD-related uveitis, providing a long-term control of ocular inflammation.

Disclosure of Interest: None declared

DOI: 10.1136/annrheumdis-2017-eular.1026

\section{THU0541 ROLE OF IL-1 INHIBITION IN ADULT ONSET STILL'S DISEASE (AOSD): A RETROSPECTIVE, OBSERVATIONAL MULTICENTRIC STUDY FROM THE ITALIAN SOCIETY OF RHEUMATOLOGY STUDY GROUP ON AUTOINFLAMMATORY DISEASES}

S. Colafrancesco ${ }^{1}$, R. Priori ${ }^{1}$, P. Sfriso ${ }^{2}$, G. Valesini ${ }^{1}$, L. Punzi ${ }^{2}$, M. Galeazzi ${ }^{3}$, L. Cantarini ${ }^{3}$ on behalf of The Study Group on Autoinflammatory diseases (Italian Society of Rheumatology). ${ }^{1}$ Dipartimento di medicina interna e specialità mediche, Sapienza University of Rome, Rome, ${ }^{2}$ Department of Medicine DIMED, University of Padova, Padova; ${ }^{3}$ Department of Medical Sciences, Surgery and Neurosciences, University of Siena, Siena, Italy

Background: IL-1 pathway plays a major role in the pathogenesis of Adult onset Still's disease (AOSD). IL-1 inhibitors [Anakinra (ANK) and Canakinumab (CAN)] proved their efficacy in AOSD as demonstrated in several case-reports and small case-series. However, due to the disease rarity, large randomized control trials are still lacking.

Objectives: To retrospectively evaluate the efficacy and safety of the use of IL-1 inhibitors (ANK and CAN) in patients with AOSD.

Methods: Data from patients with diagnosis of AOSD (Yamaguchi criteria) referred by 18 different Italian centres were retrospectively collected. Clinical and serological features as well as concomitant treatments, were collected on a dedicated database at the beginning of anti-IL-1 treatment (baseline) and then after 3, 6 and 12 months of follow-up. The Pouchot's score was used to evaluate disease activity.

Results: Data from 140 patients were collected (Table). All were treated with ANK and 4 were subsequently switched to CAN after ANK failure. The systemic pattern vs the chronic-articular were present in ANK and CAN groups in $104 / 140(74.2 \%)$ and $3 / 4(75 \%)$, respectively, vs $48 / 140(25.8 \%)$ and $1 / 4(25 \%)$, respectively. MTX was the most commonly used DMARD before ANK or CAN [91/140 (75.8\%), 2/4 (50\%) respectively]. In ANK group, the most commonly used previous biologic drug (bDMARD) was etanercept (79.3\%). ANK was adopted as second-line bDMARD in 29/140 (20.7\%) patients. In most of the cases it was administered at $100 \mathrm{mg} /$ day in $126 / 140$ patients (90\%). CAN was employed at $150 \mathrm{mg}$ every 8 weeks. ANK was effective on all AOSD clinical (fever, rash, pneumonia, pericarditis, pleuritis, sorethroat, lymphadenopathy, hepatomegaly, myalgia, arthritis, MAS) and serological (increased liver enzymes, hyperferritinemia, leucocytosis) manifestations $(p<0.0001)$. The Pouchot's score was significantly reduced at all time points $(p<0.0001)$. In ANK group no difference in treatment response was identified stratifying patients according to age, sex, pattern of disease, monotherapy vs combination. ANK primary and secondary inefficacy after 12 months were 15/140 (10.7\%) and 11/140 (7.8\%) cases, respectively. Adverse events (AEs) [mainly represented by in situ $(28 / 47,59.5 \%)$ or diffused $(12 / 47,25.5 \%)$ skin reactions and infections (12/47, 12.7\%)] were the main reason for ANK discontinuation. Similarly, in CAN group Pouchot's score and clinical and serological features significantly ameliorated at all time points

\begin{tabular}{|c|c|c|}
\hline Clinical features & ANK group & CAN group \\
\hline $\operatorname{sex}(M / F)$ & $47 / 93$ & $0 / 4$ \\
\hline Age at onset (mean $\pm S D$, years) & $35.4 \pm 17$ & $34.7 \pm 13.3$ \\
\hline Age at diagnosis (me an $\pm S D$, years) & \multirow{2}{*}{$37.4 \pm 16.1$} & $34.2 \pm 15.4$ \\
\hline Dise ase duration before tre atment (me an $\pm \mathrm{SD}$, months) & & $58.33 \pm 48.4$ \\
\hline Previoustherapies: & \multirow{2}{*}{$137 / 140(97.8 \%)$} & $4 / 4(100 \%)$ \\
\hline -STEROIDS & & \multirow{3}{*}{$\begin{array}{l}2 / 4(50 \%) \\
2 / 4(50 \%)\end{array}$} \\
\hline - DMARDS & \multirow{2}{*}{$\begin{array}{l}120 / 140(85.7 \%) \\
29 / 140(20.7 \%)\end{array}$} & \\
\hline - DDMARDS & & \\
\hline Monotherapy & $34 / 140(24.2 \%)$ & $2 / 4(50 \%)$ \\
\hline Combination therapy (-DMARDs) & $106 / 140(75.8 \%)$ & $2 / 4(50 \%)$ \\
\hline$N^{0}$ Patient baseline & $140(100 \%)$ & $4 / 4(100 \%)$ \\
\hline $\mathrm{N}^{0}$ patients 3 months & $118(84.2 \%)$ & $4 / 4(100 \%)$ \\
\hline$N^{0}$ patients 6 months & $109(77.8 \%)$ & $4 / 4(100 \%)$ \\
\hline$N^{0}$ patients 12 months & $97(69.2 \%)$ & $3 / 4(75 \%)$ \\
\hline $\mathrm{N}^{\circ}$ patients at the time of this study & $69(49.2 \%)$ & $2 / 4(50 \%)$ \\
\hline Me an duration of therapy (mean $\pm S D$, months) & $35.7 \pm 36.1$ & $22.1 \pm 16.5$ \\
\hline AES & $47 / 140(33.5 \%)$ & $0 / 4(0 \%)$ \\
\hline \multicolumn{3}{|l|}{ Reasons of discontinuation: } \\
\hline -AEs & $24 / 71(33.8 \%)$ & $0 / 4(0 \%)$ \\
\hline -Remission & $20 / 71(28.1 \%)$ & $1 / 4(25 \%)$ \\
\hline -Primary inefficacy & $16 / 71(22.5 \%)$ & $0 / 4(0 \%)$ \\
\hline -Loss of efficacy & $11 / 71(15.4 \%)$ & $1 / 4(25 \%)$ \\
\hline Pouchot's score at baseline & $5.5 \pm 1.9$ & $4.2 \pm 2.6$ \\
\hline Pouchots score at 3 months & $1.1 \pm 1.4$ & $1.2 \pm 1.8$ \\
\hline Pouchots score at 6 months & $0.6 \pm 1.2$ & $1.5 \pm 1.9$ \\
\hline Pouchot's score at 12 months & $0.4 \pm 0.8$ & $1 \pm 1.4$ \\
\hline$\%$ patients with steroids (baseline) & $137 / 140(97.8 \%)$ & $4 / 4(100 \%)$ \\
\hline$\%$ patients with steroids (time of the study) & $22 / 69(31.8 \%)$ & $2 / 2(100 \%)$ \\
\hline$\%$ patients with DMARDs (base line) & $120 / 140(85.7 \%)$ & $2 / 4(50 \%)$ \\
\hline$\%$ patients with DMARDs (time of the study) & $35 / 69(50.7 \%)$ & $0 / 2(0 \%)$ \\
\hline
\end{tabular}


$(p<0.0001)$. No AEs occurred with CAN, only in one case $(1 / 4,25 \%)$ treatment was stopped for loss of efficacy.

Conclusions: To our knowledge this is the largest retrospective observational study evaluating the efficacy and safety of ANK and CAN in AOSD. A prompt response was demonstrated already at 3 months of follow-up both in ANK and CAN groups. ANK proved to be effective in both patterns of disease, appearing also safe on the infectious risk. Nonetheless, skin reaction may represent a not negligible AEs during ANK treatment.

Disclosure of Interest: None declared

DOI: 10.1136/annrheumdis-2017-eular.6166

\section{THU0542 ANTI-INTERLEUKIN 1 THERAPY IN FMF AMYLOIDOSIS: A SINGLE CENTER EXPERIENCE (CASE SERIES)}

S. Ugurlu, B. Ergezen, A. Hacioglu, H. Ozdogan. Division of Rheumatology, Department of Internal Medicine, Cerrahpasa Medical Faculty, University of Istanbul, Istanbul, Turkey

Background: Recently there is increasing number of reports investigating the efficacy of anti-interleukin-1 (anti-IL1) therapy in AA-amyloidosis.

Objectives: Here we report our experience in $\mathrm{IL}-1$ blockade in $\mathrm{AA}$ amyloidosis secondary to FMF.

Methods: Twenty nine FMF patients with secondary AA-amyloidosis with insufficient response to colchicine were treated with anti-IL-1 agents (canakinumab and anakinra). Creatinine (Cre), 24-hour urine protein (UP), erythrocyte sedimentation rate $(E S R)$ and $C$-reactive protein $(C R P)$ were measured before and throughout the treatment to evaluate the response and side effects.

Results: Twenty nine (16 female, 13 male) patients with FMF-related amyloidosis were administered anti-IL1 agents (12 on canakinumab, 17 on anakinra) in addition to colchicine in 27 patients, with a mean dose of $1.4 \pm 0.6 \mathrm{mg} /$ day. The mean age was $40.13 \pm 11.76$, while the mean duration of FMF was $28.79 \pm 10.51$ years. The mean follow-up was $13.92 \pm 11.31$ months for anakinra and $11.82 \pm 9.92$ months for canakinumab.

Initial Cre levels were less than $1.5 \mathrm{mg} / \mathrm{dl}$ in 13 patients (range 0.37-.5). In this subgroup proteinuria decreased significantly from $3739.87 \pm 4860.41$ to $1321.45 \pm 2015.62 \mathrm{mg} / \mathrm{day}$ while Cre was stable $(0.91 \pm 0.30 \mathrm{mg} / \mathrm{dl}$ to $1.04 \pm 0.39$ $\mathrm{mg} / \mathrm{dl}$ ) and acute phase response was normalized (CRP from $6.16 \pm 7.86 \mathrm{mg} / \mathrm{l}$ to $5.20 \pm 9.64 \mathrm{mg} / \mathrm{l}$, ESR from $27.3 \pm 18.63$ to $14 \pm 8.04)$.In the second group there were 11 patients with initial Cre levels higher than $1.5 \mathrm{mg} / \mathrm{dl}$ (range 1.73-3.76). Proteinuria decreased from $6321.66 \pm 5936.43$ to $4827.55 \pm 6264.43 \mathrm{mg} /$ day, Cre increased from $2.53 \pm 0.76$ to $3.07 \pm 1.70 \mathrm{mg} / \mathrm{dl}$, while there was a decrease in the APR in this subgorup (CRP from $40 \pm 64.65 \mathrm{mg} / \mathrm{l}$ to $26.71 \pm 26.76 \mathrm{mg} / \mathrm{l}$, ESR from $57.37 \pm 37.65$ to $39.11 \pm 27.72$ ).

There are two patients on hemodialysis whereas two underwent renal transplantation. Global patient assessment score of the whole group was decreased from $7.44 \pm 2.54$ to $3.89 \pm 3.53$ with anti-IL-1

Anakinra was stopped in 11 and canakinumab in 3 patients, due to irresponsiveness in 8 and 2 patients respectively. Among patients in whom anakinra was terminated 9 were later treated with canakinumab. Twenty four patients are still recieving anti-IL-1treatment (16 on anakinra, 8 on canakinumab).

Conclusions: Anti IL-1 treatments seems to be effective and safe in the treatment of AA amyloidosis secondary to FMF. The beneficial effect of this approach is more pronounced in patients with creatinine levels of less that $1.5 \mathrm{mg} / \mathrm{dl}$.

Disclosure of Interest: None declared

DOI: 10.1136/annrheumdis-2017-eular.5397

\section{THU0543 A RETROSPECTIVE OVERVIEW OF THE TREATMENT MODALITY, OUTCOME AND RELAPSE RISK OF IGG4 RELATED DISEASE IN HONG KONG: A DATASET OF 108 PATIENTS FROM FOUR REGIONAL HOSPITALS}

C.P. Tang ${ }^{1}$, K.L. Lee ${ }^{1}$, K.W. Lee ${ }^{2}$, W.L. $\mathrm{Ng}^{3}$, M.C. Wan ${ }^{4}$, K.Y. Yuen ${ }^{5}$. ${ }^{1}$ Medicine, PYNEH; ${ }^{2}$ Medicine, HKSH; ${ }^{3}$ Medicine, UCH; ${ }^{4}$ Medicine, $\mathrm{RH} ;{ }^{5}$ Medicine, QMH, HK, Hong Kong

Background: Currently most consensus suggests treating lgG4 related disease (lgG4RD) with prednisolone $0.6 \mathrm{mg} / \mathrm{kg} /$ day tapering over 6 months while some experts prefer continuation of steroid up to 3 years $(1-3)$.

Objectives: In this retrospective study, the treatment modality, response and relapse risk of IgG4RD patients over the past ten years from four regional hospitals in Hong Kong were analyzed.

Methods: Four regional hospitals participated with study period from 1/1/2006 to $30 / 6 / 2016$. Patients were diagnosed IgG4RD according to the Japanese Comprehensive Diagnostic Criteria for IgG4RD. Treatment response at 6 months was recorded as complete, partial or non-remission based on the patient's and physician's perspective. Relapse was defined as disease progression either clinically or radiologically

Statistical analysis: The association between treatment response and steroid dosage was analyzed by multivariate logistic regression. Risk factors for relapse were analyzed by multivariate cox regression and the hazard ratio was reported. Results: 37 cases had surgical excision and disease recurred in 5 cases (relapse rate $=13.5 \%$ ). 87 patients $(81 \%)$ received steroid treatment. The mean starting prednisolone was $33.5 \mathrm{mg}$ daily, with a mean duration of 95.2 weeks. At 6 months,
5 patients $(6 \%)$ had no response, 34 patients $(41 \%)$ had partial remission and 44 patients $(53 \%)$ had complete remission.

29 patients also received other immunosuppressants, including azathioprine $(n=25)$, mycophenolate mofetil (MMF) $(n=6)$, cyclosporine $A(n=4)$, mercaptopurine $(n=2)$ and rituximab $(n=1)$. The overall response rate for azathioprine and MMF was $64 \%$ and $67 \%$ respectively.

In the final multiple logistic regression, an initial prednisolone $30 \mathrm{mg}$ daily or above was associated with a higher complete remission at 6 months $(O R=3.4, p=0.079)$ and the effect was more seen in patients with salivary and orbital involvement $(\mathrm{OR}=6.8, \mathrm{p}=0.10)$

18 patients relapsed after steroid was stopped and 6 patients relapsed while on prednisolone $2.5-7.5 \mathrm{mg}$ daily. The one year, two year and three year relapse rate were $13.3 \%, 24.1 \%$ and $26.5 \%$ respectively.

In the final multivariate cox regression, the presence of maintenance steroid was associated with a lower relapse risk (Hazard ratio $=0.121, p=0.000$ ) while serum IgG4 level above twice upper limit of normal (Hazard ratio $=5.283, p=0.029$ ) and hepatobiliary involvement (Hazard ratio $=2.164, \mathrm{p}=0.095$ ) were associated with a higher relapse risk.

Conclusions: Most patients (94\%) had good response to steroid. Patients with hepatobiliary involvement and serum IgG4 level above twice upper limit of normal were at higher relapse risk and a low dose, maintenance prednisolone for longer period is recommended.

References:

[1] Kleger A, et al. IgG4-related autoimmune diseases: Polymorphous presentation complicates diagnosis and treatment. Deutsches Arzteblatt international. 2015;112(8):128-35

[2] Ghazale A, et al. Immunoglobulin G4-associated cholangitis: clinical profile and response to therapy. Gastroenterology. 2008;134(3):706-15.

[3] Kamisawa $T$, et al. Standard steroid treatment for autoimmune pancreatitis. Gut. 2009;58(11):1504-7.

Disclosure of Interest: None declared

DOI: 10.1136/annrheumdis-2017-eular.2154

\section{THU0544 THE IMPORTANCE OF R202Q POLYMORPHISM IN CLINICAL EXPRESSION OF FMF: A SINGLE CENTER CROSS-SECTIONAL STUDY}

S. Yilmaz ${ }^{1}$, E. Tekgoz ${ }^{1}$, F.I. Cinar ${ }^{2}$, M. Cinar ${ }^{1}$. ${ }^{1}$ Department of Internal Medicine, Division of Rheumatology, University of Health Sciences, Gulhane Medical Faculty; ${ }^{2}$ Gulhane School of Nursing, University of Health Sciences, Ankara, Turkey

Background: Familial Mediterranean fever (FMF) is an autosomal recessive inherited disease with recurrent fever and inflammatory episodes of serous membranes. The MEFV (Mediterranean fever) gene in the short arm of chromosome 16 is affected in the FMF. This gene encodes for a protein called 'Pyrin'. The erroneously synthesized Pyrin protein due to MEFV mutations is unable to control the post inflammatory process. Although there have been many efforts to find out genotype-phenotype association in FMF, no clear relationship has been clarified.

Objectives: In this study, the relationship between FMF clinical symptoms and MEFV gene mutations and polymorphism was investigated.

Methods: Total of 158 patients with FMF was included to the study that was conducted in a tertiary rheumatology outpatient clinic. The demographic and clinical features, as well as MEFV gene mutations were recorded in a "Patient Assessment Form". The clinical status of the disease was evaluated with FMFseverity score-2 (F-SS-2). The associations between clinical features and genetic alterations were calculated with Pearson Chi-square test.

Results: The mean age of patients was $24.3 \pm 5.1$, mean delay in diagnosis was $5.6 \pm 6.3$ years, and 155 of the patients (\%98.1) were male. The percentage of patients stating they use colchicine regularly was $136(86.1 \%)$, and mean dose was $1.4 \pm 0.3 \mathrm{mg} /$ day. The most frequent mutation was M694V $(76.6 \%)$, and $\mathrm{R} 202 \mathrm{Q}, \mathrm{M} 680 \mathrm{I}$ and $\mathrm{E} 148 \mathrm{Q}$ were found in a descending order $(60.8 \%, 19.0 \%$ and $13.9 \%$, respectively). The FMF severity score was found to be higher in patients carrying M694V mutation ( $\mathrm{p}=0.01$ ). In comparison with negative family history for FMF, M694V was more prevalent in patients with positive family history $(82.0 \%$ vs $67.2 \%, p=0.035$ ). Besides, M694V mutation found to be associated with arthritis $(p=0.045) . E 148 Q$ mutation was associated with the history of orchitis $(p=0.029)$. The most significant relation between $\mathrm{E} 148 \mathrm{Q}$ and clinical feature was family history of hemodialysis $(\mathrm{p}=0.005)$. The prevalence of non-periodic myalgia was $\% 34.2$, and this symptom was not present in patients carrying V726A mutation $(p=0.005) . M 694 V / R 202 Q$ was the most prevalent compound heterozygosis and found in 16 patients (10.1\%). This mutation (M694V/R202Q) was associated with fewer frequencies of myalgia and peritonitis, and with good response to colchicine.

Conclusions: The presence of $\mathrm{R} 202 \mathrm{Q}$ polymorphism is associated with FMF, and should be considered in the routine genetic analysis of the disease. In our patients, its co-existence with M694V seems to be associated with good response to colchicine, and to alleviate the severity of the disease expression of M694V, which is known to be associated with severe course.

\section{References:}

[1] Mor A, Shinar Y, Zaks N, Langevitz P, Chetrit A, Shtrasburg S, Rabinovitz E, Livneh A. Evaluation of disease severity in familial Mediterranean fever. Semin Arthritis Rheum. 2005 Aug;35(1):57-64 